OPEN ACCESS

Edited by:

Songwen Tan

Central South University, China

Reviewed by:

Fang Wei,

Central South University, China

Wenjun Gu,

Shanghai Jiaotong University School of Medicine, China

*Correspondence:

Feng Yu

lianghwzj198897@163.com

Specialty section: This article was submitted to

Visceral Surgery,

a section of the journal

Frontiers in Surgery

Received: 18 January 2022

Accepted: 09 February 2022

Published: 07 March 2022

Citation:

Liang $P$ and Yu F (2022) Value of CRP,

$P C T$, and NLR in Prediction of Severity

and Prognosis of Patients With

Bloodstream Infections and Sepsis.

Front. Surg. 9:857218.

doi: 10.3389/fsurg.2022.857218

\section{Value of CRP, PCT, and NLR in Prediction of Severity and Prognosis of Patients With Bloodstream Infections and Sepsis}

\author{
Peipei Liang and Feng $\mathrm{Yu}^{*}$ \\ Department of Emergency Intensive Care Unit, The First Affiliated Hospital of AnHui Medical University, Hefei, China
}

Objective: To investigate the value of C-reactive protein (CRP), procalcitonin (PCT), and neutrophil to lymphocyte ratio (NLR) in assessing the severity of disease in patients with bloodstream infection and sepsis, and to analyze the relationship between the levels of three inflammatory factors and the prognosis of patients.

Methods: The clinical data of 146 patients with bloodstream infection and sepsis admitted to our intensive care unit (ICU) from October 2016 to May 2020 were retrospectively analyzed. The differences in the levels of inflammatory indicators such as CRP, PCT, and NLR within $24 \mathrm{~h}$ in patients with bloodstream infection sepsis with different conditions (critical group, non-critical group) and the correlation between these factors and the condition (acute physiology and chronic health evaluation II, APACHE II score) were analyzed. In addition, the prognosis of all patients within 28 days was counted, and the patients were divided into death and survival groups according to their mortality, and the risk factors affecting their death were analyzed by logistic regression, and the receiver operating characteristic $(R O C)$ curve was used to analyze the value of the relevant indicators in assessing the prognosis of patients.

Results: The levels of NLR, CRP, PCT, total bilirubin (TBIL), glutamic oxaloacetic transaminase (AST), and serum creatinine (Scr) were significantly higher in the critically ill group than in the non-critically ill group, where correlation analysis revealed a positive correlation between CRP, PCT, and NLR and APACHE II scores $(P<0.05)$. Univariate logistic regression analysis revealed that CRP, PCT, NLR, and APACHE II scores were associated with patient prognosis $(P<0.05)$. Multi-factor logistic regression analysis found that PCT, NLR, and APACHE II scores were independent risk factors for patient mortality within 28 days $(P<0.05)$. ROC curve analysis found that PCT and NLR both had an AUC area $>0.7$ in predicting patient death within 28 days $(P<0.05)$.

Conclusion: Inflammatory factors such as NLR, CRP, and PCT have important clinical applications in the assessment of the extent of disease and prognosis of patients with bloodstream infection and sepsis.

Keywords: C-reactive protein, procalcitonin, neutrophil to lymphocyte ratio, bloodstream infection and sepsis, prognosis, condition 


\section{BACKGROUND}

Bloodstream infection is a serious infectious disease that occurs when pathogenic microorganisms such as bacteria and fungi invade the blood circulation through a damaged skin mucosal barrier, causing systemic disseminated infection, and septicemia and bacteremia are often referred to clinically as bloodstream infection (1,2). Clinical practice shows (3) that patients with bloodstream infections caused by bacterial infections have a very high probability of developing sepsis, and some studies (4) suggest that patients with bacterial bloodstream infections develop rapidly and can develop septic shock within a short period of time, which can lead to multi-organ failure or even death in severe cases. The incidence of sepsis is increasing every year and has a very high mortality rate $(5,6)$. It has been shown $(7,8)$ that if patients can be treated correctly within $1 \mathrm{~h}$ of onset, their survival rate can be $80 \%$ or more, while if patients are treated more than $6 \mathrm{~h}$ after onset their probability of survival is more than $30 \%$. Thus, early risk stratification and timely identification of critical conditions are key to improving the short-term prognosis of patients with bloodstream infection sepsis.

Currently, blood cultures are the gold standard for the diagnosis of bloodstream infections, but they are timeconsuming, taking 3-7 days or more, with poor timeliness and the possibility of contaminated specimens (9). In recent years, some clinical studies have found that the levels of inflammatory factors such as calcitoninogen (PCT) and Creactive protein (CRP) have high clinical value in the early diagnosis of sepsis and determination of the disease $(10,11)$. PCT and CRP are two important inflammatory cytokines that are involved in apoptosis and can enable cell lysis, and their levels are significantly increased when the organism is infected with bacteria, but the relevance of changes in their levels has been less studied in patients with sepsis due to bloodstream infection (12). In addition, studies (13, 14) have shown that the neutrophil/lymphocyte ratio (NLR) can be used to predict sepsis bloodstream infections and help distinguish between different pathogenic species, but it is not widely accepted by clinicians as a definitive marker of infection and the exact threshold value is controversial. Therefore, this study was conducted to investigate the correlation between PCT, CRP, and NLR and the prognostic value of bloodstream infection in patients with sepsis, in order to provide a basis for early screening of high-risk patients and to guide clinical treatment.

\section{INFORMATION AND METHODS}

\section{Inclusion Criteria}

One hundred and forty-six patients with bloodstream infection sepsis (positive blood cultures) admitted to our intensive care unit (ICU) from October 2016 to May 2020 were selected, and only clinical data from the first admission were selected for repeat ICU admissions during the study period. Inclusion criteria: (1) referring to the International Consensus on the
Definition of Sepsis and Infectious Shock, Third Edition (Sepsis3) published in 2016 (15), the criteria for sepsis diagnosis were (i) identification of suspected infection; (ii) presence of a sequential organ failure score (SOFA) change $\geq 2$ points; (2) the patient's age was $\geq 18$ years; (3) the patient's case data and biochemical findings after admission were complete and free of defects; (4) two or more positive blood cultures, or two sites with the same positive blood culture and the same causative organism; (5) the patient's serum inflammatory factors PCT, CRP and NLR levels were measured within $24 \mathrm{~h}$ after admission. Exclusion criteria: (1) patients who had been hospitalized for $<24 \mathrm{~h}$; (2) patients with other viral or bacterial infections; (3) patients with immune deficiency, malignancy, hematologic disorders, or severe psychiatric disorders; (4) patients who had been treated with antibiotics prior to initial admission; (5) women who were breastfeeding or pregnant; (6) patients or family members who had requested that treatment be abandoned.

\section{General Information}

The clinical data of 146 patients with sepsis from bloodstream infection, 60 women and 86 men, aged 18-94 (62.45 \pm 14.16) years, included in the study were retrospectively analyzed. Information on gender, age, site of infection, comorbidities [multiple organ failure syndrome (MODS), acute respiratory distress syndrome (ARDS)], underlying diseases (chronic obstructive pulmonary disease, hypertension, diabetes mellitus), blood count [leucocytes (WBC), total bilirubin (TBIL), D-Dimer (D-D), Alanine transaminase (ALT), aspartate transaminase (AST), B-type natriuretic peptide (BNP), platelet count (PLT), serum creatinine (Scr)], inflammatory factor levels (CRP, PCT, and NLR), type of infectious strain, and APACHE II score at $24 \mathrm{~h}$ of admission were collected from all patients by case collection.

\section{Grouping Methods}

The Acute Physiology and Chronic Health Rating Scale (APACHE II) assessment was completed within $24 \mathrm{~h}$ after patients were admitted to the ICU, and patients were divided into a critical group (67 patients with an APACHE II score of 20 or more) and a non-critical group (79 patients with an APACHE II score of $<20$ ) based on their scores. Patients were observed starting from the time of admission to the hospital and discharged with improvement or death within 28 days as the end point. The prognosis of all patients was counted and divided into a death group (56 patients) and a survival group (90 patients) according to their prognosis. Among them, survival means patients who improved after treatment in ICU and were transferred to other departments for further treatment or discharged from the hospital; death includes patients who died after ineffective treatment and those who were automatically discharged.

\section{Statistical Processing}

All data collected were statistically analyzed and plotted using SPSS 22.0 and Prism 8.0. The $t$-test, expressed as mean \pm standard deviation $(\mathrm{x} \pm \mathrm{s})$, was used for 
measures that conformed to normal distribution and homogeneity of variance. For the count data, the chisquare test or Fisher's exact test was performed. Pearson model was used to analyze the correlation between each index and the condition. Independent risk factors affecting prognosis were analyzed by one-way and multi-way logistic regression, and the ROC curve was used to evaluate the diagnostic sensitivity, specificity, and optimal cutoff value of each index. $p<0.05$ indicated that the difference was statistically significant.

\section{RESULTS}

\section{Comparison of General Data and Laboratory Indicators of Patients With Different Conditions}

We compared the general data and laboratory indices of patients with sepsis from severe bloodstream infection with those of patients with sepsis from non-severe bloodstream infection. The results were no statistically significant differences between the two groups in terms of gender, age, pulmonary infection, abdominal infection, concurrent MODS, concurrent ARDS, underlying disease, strain of infection, WBC level, platelet count, ALT, D-D, BNP, and BUN levels $(P>0.05)$. The levels of NLR, CRP, PCT, TBIL, AST, and Scr were higher in patients with severe bloodstream infection and sepsis than in the non-critical group, and the differences were statistically significant $(P<$ 0.05; Table 1).

\section{Analysis of the Correlation Between Laboratory Indicators and the Degree of Sepsis in Bloodstream Infections}

Pearson correlation analysis showed that NLR, CRP and PCT levels were positively correlated with severity of disease in patients with bloodstream infection sepsis ( $r$-values were 0.468 , 0.456 , and 0.670 , respectively; all $P<0.001$ ), while TBIL, AST, and Scr levels were not linearly correlated with severity of disease in patients with bloodstream infection sepsis ( $r$-values were $0.017,0.101$, and $0.117, p$-values were $0.838,0.223$, and 0.159 , respectively; Figures $\mathbf{1 A - F}$ ).

\section{Comparison of General Data and Laboratory Indicators of Patients With Different Prognosis}

We compared the general data and laboratory indices of patients in the death group with those in the survival group. The results were no statistically significant differences between the two groups in terms of gender, age, abdominal infection, underlying disease, strain of infection, WBC level, platelet count, ALT, DD, BNP, BUN, Scr, and TBIL levels $(P>0.05)$. The incidence of pulmonary infections, MODS and ARDS, the levels of NLR, CRP, PCT, and AST, and the APACHE II score were significantly higher in the death group than in the survival group $(P<0.05$; Table 2$)$.

TABLE 1 | Comparison of general data and laboratory indicators of patients with different conditions $[(x \pm s), n(\%)]$.

\begin{tabular}{|c|c|c|c|c|c|}
\hline \multicolumn{2}{|c|}{ Information } & Critical group $(n=67)$ & Non-critical group $(n=79)$ & $\chi^{2} / t$ value & $P$-value \\
\hline \multicolumn{2}{|c|}{ Gender (male) } & 38 (56.72) & $48(60.76)$ & 0.245 & 0.621 \\
\hline \multicolumn{2}{|c|}{ Age (years) } & $63.32 \pm 13.45$ & $61.57 \pm 15.16$ & 0.903 & 0.369 \\
\hline \multirow[t]{2}{*}{ Infection status } & Pulmonary infection & $47(70.15)$ & $62(78.48)$ & 2.094 & 0.148 \\
\hline & Abdominal infection & 10 (14.93) & 16 (20.25) & 0.703 & 0.402 \\
\hline \multicolumn{2}{|c|}{ Complicated MODS } & $10(14.93)$ & $13(16.46)$ & 0.064 & 0.800 \\
\hline & Hypertension & $22(32.84)$ & $36(45.57)$ & 2.455 & 0.117 \\
\hline & Diabetes mellitus & $8(11.94)$ & $12(15.19)$ & 0.324 & 0.569 \\
\hline \multirow[t]{2}{*}{ Types of bacteria } & Gram negative bacteria & $32(47.76)$ & 45 (56.96) & 1.231 & 0.267 \\
\hline & Gram positive bacteria & $28(41.79)$ & $41(51.90)$ & 1.486 & 0.222 \\
\hline NLR & & $27.32 \pm 16.33$ & $12.58 \pm 7.14$ & 7.250 & 0.000 \\
\hline $\operatorname{PLT}\left(\times 10^{9} / \mathrm{L}\right)$ & & $130.26 \pm 62.24$ & $113.51 \pm 52.33$ & 1.767 & 0.079 \\
\hline TBIL $(\mu \mathrm{mol} / \mathrm{L})$ & & $34.65 \pm 42.13$ & $21.83 \pm 29.54$ & 2.152 & 0.033 \\
\hline AST (U/L) & & $84.23 \pm 33.16$ & $56.77 \pm 27.56$ & 5.465 & 0.000 \\
\hline ALT (U/L) & & $37.26 \pm 13.24$ & $35.58 \pm 11.48$ & 0.821 & 0.413 \\
\hline D-D (mg/L) & & $4.24 \pm 3.10$ & $4.05 \pm 3.25$ & 0.360 & 0.720 \\
\hline BNP (ng/L) & & $3246.47 \pm 3717.52$ & $2535.24 \pm 3017.28$ & 1.276 & 0.204 \\
\hline $\mathrm{BUN}(\mathrm{mmol} / \mathrm{L})$ & & $12.59 \pm 5.78$ & $11.34 \pm 6.04$ & 1.271 & 0.206 \\
\hline $\operatorname{Scr}(\mu \mathrm{mol} / \mathrm{L})$ & & $208.13 \pm 114.58$ & $164.32 \pm 132.26$ & 2.119 & 0.036 \\
\hline
\end{tabular}




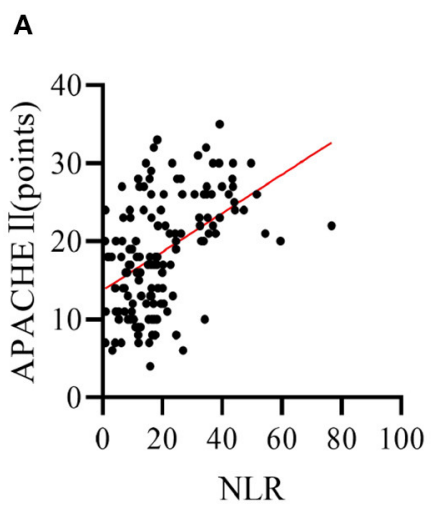

D

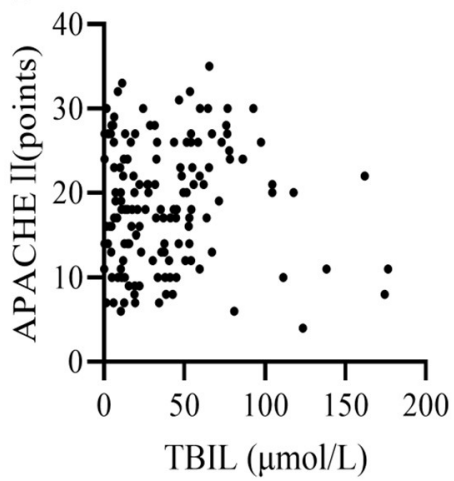

B

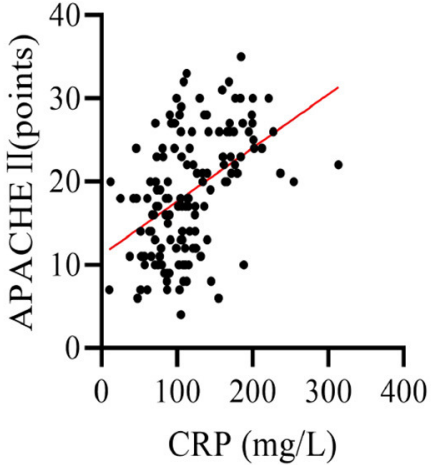

E

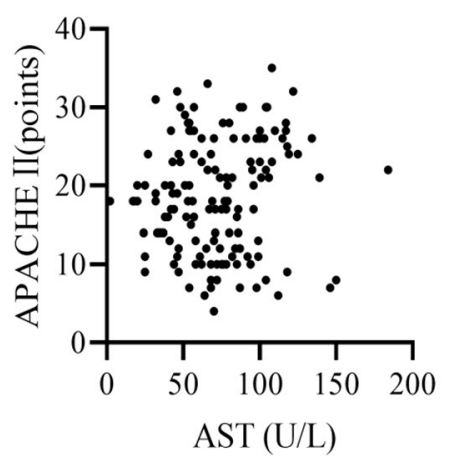

C

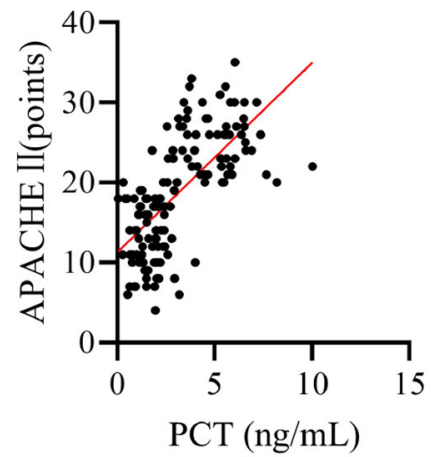

$\mathbf{F}$

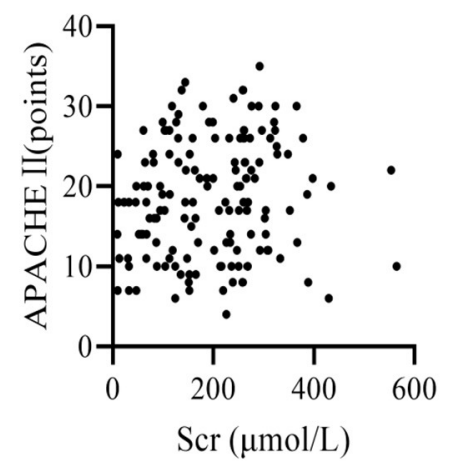

FIGURE 1 | Pearson correlation analysis showed that the levels of (A) NLR, (B) CRP, and (C) PCT were positively correlated with the severity of disease (APACHE II) in patients with bloodstream infection sepsis ( $r$ values of 0.468, 0.456, and 0.670 respectively; all $P$ values <0.001); (D) TBIL, (E) AST, and (F) SCR levels were not linearly correlated with the severity of disease in patients with bloodstream infection sepsis ( $r$ values of $0.017,0.101$, and 0.117 respectively, $P$ values of $0.838,0.223$, and 0.159 respectively).

\section{Logistic Regression Analysis of Risk Factors Affecting 28 Days Mortality in Patients With Bloodstream Infection and Sepsis}

Patients' prognosis at 28 days was used as the dependent variable ( survival $=0$, death $=1$ ) and relevant influencing factors were used as independent variables (see Table $\mathbf{3}$ for assignments) to enter a single multifactorial analysis. Logisti univariate analysis showed that NLR level, CRP level, PCT, and APACHE II score were associated with 28 days mortality in patients with bloodstream infection and sepsis $(P<0.05)$. Further multiple regression analysis of the indicators that differed in the univariate analysis showed that NLR level, PCT level and APACHE II score were independent risk factors for death at 28 days in patients with bloodstream infection and sepsis $(P<0.05$; Tables 3, 4).

\section{Analysis of the Predictive Value of Relevant Indicators on the Prognosis of Patients With Bloodstream Infection and Sepsis}

The area under the curve (AUC) for NLR, PCT, and APACHE II scores to predict 28 days death in patients with bloodstream infection and sepsis were 0.791 (95\% CI: 0.714-0.868), 0.830
(95\% CI: 0.758-0.902), and 0.718 (95\% CI: 0.636-0.800), respectively, all with $P<0.05$ (Table 5; Figure 2).

\section{DISCUSSION}

Bloodstream infection is usually defined as the same patient's blood samples obtained from different parts of the body to culture the same bacteria, and the patient shows symptoms and signs related to the pathogenic bacteria, it is a serious infectious disease that can spread throughout the body and is often lifethreatening (16). Sepsis is usually triggered by dysregulation of the organism's response to infection and is an acute organ dysfunction resulting from an abnormal immune response of the host to infection (17). Some clinical studies (18) have shown that among the various types of infections that cause sepsis, bloodstream infection is the most important factor in the death of patients with sepsis. And with the gradual increase in the incidence of sepsis after bloodstream infection in China in recent years, the research on the condition, prognosis assessment and prevention and treatment of sepsis caused by bloodstream infection has become a hot spot of concern for clinical workers.

Most scholars $(19,20)$ currently believe that the development, progression and prognosis of sepsis in bloodstream infections are 
TABLE 2 | Comparison of general data and laboratory indicators of patients with different prognosis [(x $\pm \mathrm{s}), n(\%)]$.

\begin{tabular}{|c|c|c|c|c|c|}
\hline \multicolumn{2}{|c|}{ Information } & Death group $(n=56)$ & Survival group $(n=90)$ & $\chi^{2} / \mathrm{t}$ value & $P$-value \\
\hline \multicolumn{2}{|c|}{ Gender (male) } & $36(64.29)$ & $50(55.56)$ & 0.016 & 0.900 \\
\hline \multicolumn{2}{|c|}{ Age (years) } & $60.23 \pm 17.46$ & $55.72 \pm 17.65$ & 1.507 & 0.134 \\
\hline \multirow[t]{2}{*}{ Infection status } & Pulmonary infection & $48(85.71)$ & $61(67.78)$ & 5.870 & 0.015 \\
\hline & Abdominal infection & $8(14.29)$ & $18(20.00)$ & 0.770 & 0.380 \\
\hline Complicated MODS & & $14(25.00)$ & $9(10.00)$ & 5.852 & 0.016 \\
\hline Complicated ARDS & & $12(21.43)$ & $8(8.89)$ & 4.592 & 0.032 \\
\hline \multirow[t]{3}{*}{ Underlying disease } & COPD & $6(10.71)$ & $6(6.67)$ & 1.124 & 0.289 \\
\hline & Hypertension & 19 (33.93) & $39(43.33)$ & 1.275 & 0.259 \\
\hline & Diabetes mellitus & $6(10.71)$ & $14(15.56)$ & 0.684 & 0.408 \\
\hline \multirow[t]{2}{*}{ Types of bacteria } & Gram negative bacteria & 33 (58.93) & 44 (48.89) & 2.576 & 0.109 \\
\hline & Gram positive bacteria & $23(41.07)$ & $46(51.11)$ & 1.396 & 0.237 \\
\hline NLR & & $29.37 \pm 12.24$ & $13.52 \pm 8.22$ & 9.360 & 0.000 \\
\hline CRP (mg/L) & & $150.59 \pm 57.37$ & $106.24 \pm 62.25$ & 4.312 & 0.000 \\
\hline PCT (ng/mL) & & $9.43 \pm 4.25$ & $2.56 \pm 1.61$ & 13.844 & 0.000 \\
\hline WBC $\left(\times 10^{9} / \mathrm{L}\right)$ & & $15.02 \pm 11.34$ & $12.57 \pm 9.43$ & 1.411 & 0.160 \\
\hline $\operatorname{PLT}\left(\times 10^{9} / \mathrm{L}\right)$ & & $132.88 \pm 58.42$ & $118.25 \pm 48.37$ & 1.639 & 0.103 \\
\hline TBIL ( $\mu \mathrm{mol} / \mathrm{L})$ & & $34.77 \pm 41.82$ & $23.98 \pm 31.25$ & 1.779 & 0.078 \\
\hline AST (U/L) & & $82.56 \pm 31.43$ & $58.49 \pm 30.24$ & 4.607 & 0.000 \\
\hline ALT $(U / L)$ & & $36.11 \pm 12.25$ & $36.59 \pm 13.12$ & 0.220 & 0.826 \\
\hline D-D (mg/L) & & $4.92 \pm 3.21$ & $3.89 \pm 3.41$ & 1.815 & 0.072 \\
\hline BNP (ng/L) & & $3,310.25 \pm 3,822.01$ & $2,843.53 \pm 3,452.24$ & 0.762 & 0.447 \\
\hline $\mathrm{BUN}(\mathrm{mmol} / \mathrm{L})$ & & $13.34 \pm 6.82$ & $12.25 \pm 7.27$ & 0.902 & 0.369 \\
\hline $\operatorname{Scr}(\mu \mathrm{mol} / \mathrm{L})$ & & $196.34 \pm 119.24$ & $173.25 \pm 124.58$ & 1.107 & 0.270 \\
\hline APACHE II (points) & & $25.24 \pm 5.15$ & $20.87 \pm 4.23$ & 5.578 & 0.000 \\
\hline
\end{tabular}

TABLE 3 | Assignment table.

\begin{tabular}{ll}
\hline Indicator & Assignment \\
\hline Pulmonary infection & Yes $=0, \mathrm{No}=1$ \\
Complicated MODS & Yes $=0, \mathrm{No}=1$ \\
Complicated ARDS & Yes $=0, \mathrm{No}=1$ \\
NLR & Continuous variables \\
CRP & Continuous variables \\
PCT & Continuous variables \\
AST & Continuous variables \\
APACHEII & Continuous variables \\
\hline
\end{tabular}

related to the inflammatory response and the immune function status of the body. It has been reported (21) that serum levels of PCT and CRP, two important inflammatory cytokines involved in apoptosis and capable of causing cell lysis, are significantly increased when the body is infected with bacteria. PCT, a precursor of calcitonin, is a 116-amino acid glycoprotein that can be detected as a significant increase in PCT levels in the presence of bacterial infections, but is rarely seen in viral infections and is a sensitive substance for identifying bacterial and viral infections (22). CRP is an acute phase reaction protein, mainly synthesized and released by the liver, which slowly increases to hundreds or thousands of times its normal level after $8-12 \mathrm{~h}$ when the organism is invaded by pathogenic microorganisms or stimulated
TABLE 4 | Logistic regression analysis of risk factors affecting 28 days mortality in patients with bloodstream infection and sepsis.

\begin{tabular}{|c|c|c|c|c|c|c|}
\hline \multirow[t]{2}{*}{ Indicators } & \multicolumn{3}{|c|}{ Single-factor } & \multicolumn{3}{|c|}{ Multi-factor } \\
\hline & OR & $95 \% \mathrm{Cl}$ & $P$-value & OR & $95 \% \mathrm{Cl}$ & $P$-value \\
\hline $\begin{array}{l}\text { Pulmonary } \\
\text { infection }\end{array}$ & 1.379 & $0.799 \sim 2.377$ & 0.623 & - & - & - \\
\hline $\begin{array}{l}\text { Complicated } \\
\text { MODS }\end{array}$ & 1.525 & $0.695 \sim 3.347$ & 0.512 & - & - & - \\
\hline $\begin{array}{l}\text { Complicated } \\
\text { ARDS }\end{array}$ & 1.511 & $0.746 \sim 3.049$ & 0.324 & - & - & - \\
\hline NLR & 1.923 & $1.575 \sim 2.349$ & 0.012 & 1.669 & $1.402 \sim 1.987$ & 0.009 \\
\hline CRP & 1.958 & $1.030 \sim 3.724$ & 0.046 & 1.060 & $0.957 \sim 1.173$ & 0.206 \\
\hline РCT & 1.684 & $1.347 \sim 2.105$ & 0.038 & 1.687 & $1.312 \sim 2.151$ & 0.031 \\
\hline AST & 1.132 & $0.934 \sim 1.372$ & 0.344 & - & - & - \\
\hline APACHE ॥ & 2.387 & $1.223 \sim 4.657$ & 0.006 & 2.270 & $1.237 \sim 4.169$ & 0.007 \\
\hline
\end{tabular}

by inflammation, and gradually returns to normal level after reasonable treatment, which can reflect the severity of infection to some extent (23). As for the relationship between NLR and sepsis, some studies (24) have suggested that leukocytosis and lymphopenia are a distinctive feature of severe sepsis, and some foreign scholars $(25,26)$ have suggested that NLR can be used as a marker for the early detection and diagnosis of sepsis and that NLR is more effective than conventional inflammatory 
TABLE 5 | Analysis of the predictive value of relevant indicators on the prognosis of patients with bloodstream infection and sepsis.

\begin{tabular}{lccccc}
\hline Indicators & AUC & 95\% Cl & Cut-off & Sensitivity (\%) & Specificity (\%) \\
\hline NLR & 0.791 & $0.714 \sim 0.868$ & 0.476 & 64.30 & 83.30 \\
PCT & 0.830 & $0.758 \sim 0.902$ & 0.639 & 83.90 & 80.00 \\
APACHE II & 0.718 & $0.636 \sim 0.800$ & 0.326 & 89.30 & 43.30
\end{tabular}

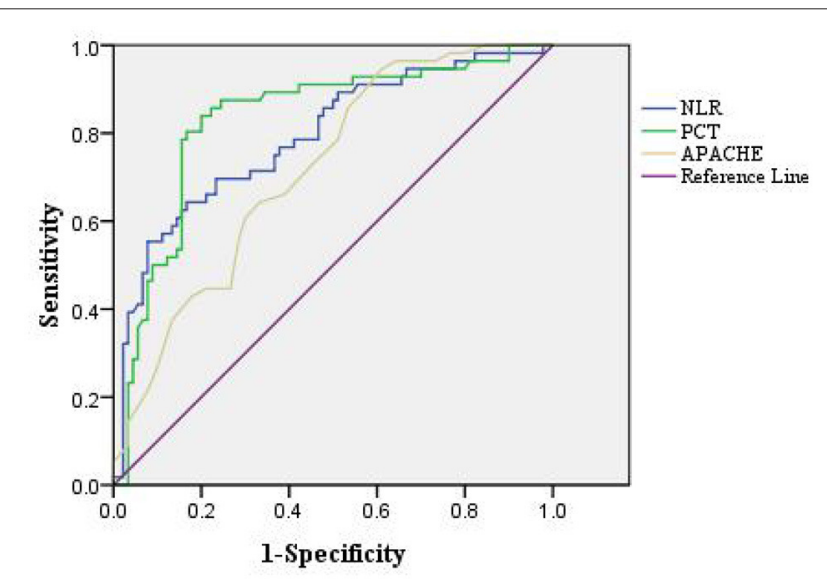

FIGURE 2 | ROC curve of the predictive value of relevant indicators for 28 days death in patients with bloodstream infection and sepsis.

biomarkers. This study focused on analyzing the differences in the levels of PCT, CRP, and NLR in patients with bloodstream infection sepsis and correlating them with the concurrently rated APACHEII to provide a reference basis for early clinical assessment of the severity of sepsis patients and their prognosis.

By evaluating the value of PCT, CRP, and NLR in assessing the condition of patients with sepsis due to bloodstream infection, it was found that the levels of NLR, CRP, and PCT were significantly higher in patients with sepsis due to severe bloodstream infection than in the non-severe group. APACHE II is currently the most commonly used disease severity scoring system in ICU, and to some extent the magnitude of its score can indicate the prognosis

\section{REFERENCES}

1. Kern WV, Rieg S. Burden of bacterial bloodstream infection-a brief update on epidemiology and significance of multidrug-resistant pathogens. Clin Microbiol Infect. (2020) 26:151-7. doi: 10.1016/j.cmi.2019. 10.031

2. Ray-Barruel G, Xu H, Marsh N, Cooke M, Rickard CM. Effectiveness of insertion and maintenance bundles in preventing peripheral intravenous catheter-related complications and bloodstream infection in hospital patients: a systematic review. Infect Dis Health. (2019) 24:152-68. doi: 10.1016/j.idh.2019.03.001

3. Torres MJM, Peterson JM, Wolf SE. Detection of infection and sepsis in burns. Surg Infect (Larchmt). (2021) 22:20-7. doi: 10.1089/sur.2020.348

4. Kawaguchi N, Katsube T, Echols R, Wajima T. Population pharmacokinetic and pharmacokinetic/pharmacodynamic analyses of cefiderocol, a parenteral siderophore cephalosporin, in patients with pneumonia, bloodstream of the disease, with the higher the score the worse the prognosis (27). Therefore, this study further correlated the inflammatory factors PCT, CRP, and NLR with APACHE II scores and found that there was a positive correlation between serum PCT, CRP, and NLR and APACHE II scores. This suggests that the severity of the disease in patients with bloodstream infection sepsis can be effectively reflected by the inflammatory factors PCT, CRP, and NLR. Meanwhile, we compared patients with different prognosis of bloodstream infection sepsis and found that higher levels of PCT and NLR tended to suggest higher sepsis mortality, and both had comparable predictive value for 28 days death in patients with bloodstream infection and sepsis.

In conclusion, NLR, CRP, and PCT can effectively reflect the severity of patients with bloodstream infection sepsis, and higher levels of NLR, CRP, and PCT are associated with severe disease. In addition NLR, PCT, and APACHE II scores are independent risk factors for predicting death in 28 days of bloodstream infection sepsis, and early monitoring of inflammatory factors for patients with bloodstream infection sepsis can be used as a marker for assessing the extent of patients' disease as well as their prognosis.

\section{DATA AVAILABILITY STATEMENT}

The original contributions presented in the study are included in the article/supplementary material, further inquiries can be directed to the corresponding author/s.

\section{ETHICS STATEMENT}

The studies involving human participants were reviewed and approved by the Ethics Committee of The First Affiliated Hospital of AnHui Medical University. The patients/participants provided their written informed consent to participate in this study.

\section{AUTHOR CONTRIBUTIONS}

FY was the supervisor of this study. All authors of this study made equal contributions, including the design of the study, the conduct of the experiments, and the writing of the paper.

infection/sepsis, or complicated urinary tract infection. Antimicrob Agents Chemother. (2021) 65:e01437-20. doi: 10.1128/AAC.01437-20

5. Font MD, Thyagarajan B, Khanna AK. Sepsis and Septic Shock Basics of diagnosis, pathophysiology and clinical decision making. Med Clin North Am. (2020) 104:573-85. doi: 10.1016/j.mcna.2020. 02.011

6. Opal SM, Wittebole X. Biomarkers of Infection and Sepsis. Crit Care Clin. (2020) 36:11-22. doi: 10.1016/j.ccc.2019.08.002

7. Fleuren LM, Klausch TLT, Zwager CL, Schoonmade LJ, Guo T, Roggeveen LF, et al. Machine learning for the prediction of sepsis: a systematic review and meta-analysis of diagnostic test accuracy. Intensive Care Med. (2020) 46:383-400. doi: 10.1007/s00134-019-05872-y

8. Jones TW, Smith SE, Van Tuyl JS, Newsome AS. Sepsis with preexisting heart failure: management of confounding clinical features. J Intensive Care Med. (2021) 36:989-1012. doi: 10.1177/0885066620 928299 
9. Greninger AL, Naccache SN. Metagenomics to assist in the diagnosis of bloodstream infection. J Appl Lab Med. (2019) 3:643-53. doi: 10.1373/jalm.2018.026120

10. Sui YD, Xin WN, Feng LL. Comparison of the clinical application values of PCT, hs-CRP and SAA detection in the early diagnosis of sepsis. Pak J Med Sci. (2020) 36:1683-7. doi: 10.12669/pjms.36.7.2544

11. Shen W, Lai S. Diagnostic Value of sCD163 Combined with PCT and HS-CRP in patients with gynecological malignant tumors and fever. J Coll Physicians Surg Pak. (2020) 30:1053-7. doi: 10.29271/jcpsp.2020.10.1053

12. Xu YB, Ouyang Y, Zhao D. Curative effects of vancomycin and cefotaxime combined with gamma globulin respectively in neonatal septicemia and their influences on PCT, CRP and hs-CRP. Eur Rev Med Pharmacol Sci. (2020) 24:4486-94. doi: 10.26355/eurrev_202004_21031

13. Huang Z, Fu Z, Huang W, Huang K. Prognostic value of neutrophil-tolymphocyte ratio in sepsis: a meta-analysis. Am J Emerg Med. (2020) 38:6417. doi: 10.1016/j.ajem.2019.10.023

14. Zhong X, Ma A, Zhang Z, Liu Y, Liang G. Neutrophil-to-lymphocyte ratio as a predictive marker for severe pediatric sepsis. Transl Pediatr. (2021) 10:657-65. doi: $10.21037 / \mathrm{tp}-21-47$

15. Singer M, Deutschman CS, Seymour CW, Shankar-Hari M, Annane D, Bauer $\mathrm{M}$, et al. The Third international consensus definitions for sepsis and septic shock (sepsis-3). JAMA. (2016) 315:801-10. doi: 10.1001/jama.2016.0287

16. Carvalho AS, Lagana D, Catford J, Shaw D, Bak N. Bloodstream infections in neutropenic patients with haematological malignancies. Infect Dis Health. (2020) 25:22-9. doi: 10.1016/j.idh.2019.08.006

17. Pierrakos C, Velissaris D, Bisdorff M, Marshall JC, Vincent JL. Biomarkers of sepsis: time for a reappraisal. Crit Care. (2020) 24:287. doi: 10.1186/s13054-020-02993-5

18. Liljedahl Prytz K, Prag M, Fredlund H, Magnuson A, Sundqvist M, Källman J. Antibiotic treatment with one single dose of gentamicin at admittance in addition to a $\beta$-lactam antibiotic in the treatment of community-acquired bloodstream infection with sepsis. PLoS One. (2020) 15:e0236864. doi: 10.1371/journal.pone.0236864

19. Chou EH, Mann S, Hsu TC, Hsu WT, Liu CC, Bhakta T, et al. Incidence, trends, and outcomes of infection sites among hospitalizations of sepsis: a nationwide study. PLoS One. (2020) 15:e0227752. doi: 10.1371/journal.pone.0227752

20. Lenglet A, Schuurmans J, Ariti C, Borgundvaag E, Charles K, Badjo C, et al. Rectal screening displays high negative predictive value for bloodstream infection with (ESBL-producing) Gram-negative bacteria in neonates with suspected sepsis in a low-resource setting neonatal care unit. J Glob Antimicrob Resist. (2020) 23:102-7. doi: 10.1016/j.jgar.2020.08.017

21. Papafilippou L, Claxton A, Dark P, Kostarelos K, Hadjidemetriou M. Nanotools for sepsis diagnosis and treatment. Adv Healthc Mater. (2021) 10:e2001378. doi: 10.1002/adhm.202001378
22. Stocker M, van Herk W, El Helou S, Dutta S, Schuerman FABA, van den Tooren-de Groot RK, et al. C-Reactive Protein, Procalcitonin, and White Blood Count to Rule Out Neonatal Early-onset Sepsis Within 36 Hours: A Secondary Analysis of the Neonatal Procalcitonin Intervention Study. Clin Infect Dis. (2021) 73:e383-90. doi: 10.1093/cid/cia a876

23. Nurmi AM, Mustonen HK, Stenman UH, Seppänen HE, Haglund CH. Combining CRP and CA19-9 in a novel prognostic score in pancreatic ductal adenocarcinoma. Sci Rep. (2021) 11:781. doi: 10.1038/s41598-020-8 0778-0

24. Root-Bernstein R. Innate receptor activation patterns involving TLR and NLR synergisms in COVID-19, ALI/ARDS and sepsis cytokine storms: a review and model making novel predictions and therapeutic suggestions. Int J Mol Sci. (2021) 22:2108. doi: 10.3390/ijms2204 2108

25. Li T, Dong G, Zhang M, Xu Z, Hu Y, Xie B, et al. Association of neutrophillymphocyte ratio and the presence of neonatal sepsis. J Immunol Res. (2020) 2020:7650713. doi: 10.1155/2020/7650713

26. Lorente L, Martín MM, Ortiz-López R, Alvarez-Castillo A, Ruiz C, Uribe L, et al. Association between neutrophil-to-lymphocyte ratio in the first seven days of sepsis and mortality. Enferm Infecc Microbiol Clin (Engl Ed). (2020). doi: 10.1016/j.eimc.2020. 11.004. [Epub ahead of print].

27. Kuo WK, Hua CC, Yu CC, Liu YC, Huang CY. The cancer control status and APACHE II score are prognostic factors for critically ill patients with cancer and sepsis. J Formos Med Assoc. (2020) 119:27681. doi: 10.1016/j.jfma.2019.05.012

Conflict of Interest: The authors declare that the research was conducted in the absence of any commercial or financial relationships that could be construed as a potential conflict of interest.

Publisher's Note: All claims expressed in this article are solely those of the authors and do not necessarily represent those of their affiliated organizations, or those of the publisher, the editors and the reviewers. Any product that may be evaluated in this article, or claim that may be made by its manufacturer, is not guaranteed or endorsed by the publisher.

Copyright (C) 2022 Liang and Yu. This is an open-access article distributed under the terms of the Creative Commons Attribution License (CC BY). The use, distribution or reproduction in other forums is permitted, provided the original author(s) and the copyright owner(s) are credited and that the original publication in this journal is cited, in accordance with accepted academic practice. No use, distribution or reproduction is permitted which does not comply with these terms. 